\title{
Establishment of geology-engineering integration model for shale gas of Wei AH platform in Weiyuan Area
}

\author{
Hai Jiang ${ }^{1, *}$, Yang Xiao ${ }^{2,3}$, Ziping Liu ${ }^{1}$, Zhigang Li $^{2}$, Xiang Deng ${ }^{1}$ and Longqing Zou ${ }^{4}$ \\ ${ }^{1}$ Shale Gas Exploration and Development Project Management Department of CNPC Chuanqing Drilling Engineering Co., LTD, \\ Chengdu, China \\ ${ }^{2}$ School of Energy, Chengdu University of Technology, Chengdu, China \\ ${ }^{3}$ Chengdu Institute of Technology Sunshine Energy Technology Co., Ltd., Chengdu, China \\ ${ }^{4}$ Downhole Operation Company of CNPC Chuanqing Drilling Engineering Co., LTD, Chengdu, China
}

\begin{abstract}
Wei AH platform is a platform composed of 8 horizontal wells in Weiyuan shale gas area. The number of faults in the area is small, and natural fractures are locally developed. Therefore, it is necessary to take volume reconstruction as the core mining technology and establish the geology-engineering integration model for the platform according to the geology-engineering integration concept. On the basis of geological model, combined with one-dimensional rock mechanics profile, the geomechanical model of Wei AH platform is established by means of deep diagenetic theory. Based on geological model and geomechanical model, the well trajectory design model, fracturing design model and reservoir numerical simulation model are modeled and optimized respectively. Finally, it can realize the comprehensive application of geophysical, geological, mechanical and seepage data, and complete the mutual call and matching of all kinds of understanding and models.
\end{abstract}

Key words: Wei AH platform; Shale gas; Geology-engineering integration; Geomechanical model; Fracturing design model.

\section{Introduction}

Weiyuan Structure is located in the middle Sichuan gulong flat tectonic zone, Weiyuan to Longnvsi tectonic group [1]. The surface and abdomen structure of well Wei A area is consistent with simple structure, high in northwest and low in southeast, and the axis is near eastwest. Wei AH platform is located in the west of well Wei A, where the number and scale of faults are small, and natural fractures developed locally. In order to realize the development of geology-engineering integration in Weiyuan shale gas field, a new connotation of geologyengineering integration with the core technical concept of "volume transformation" is put forward, and the geologyengineering integration model of the field is established [2].

More and more cases about oil and gas exploration and development showed that the technology of geologyengineering integration was an important means to realize the effective exploration and development of complex oil and gas reservoirs [3]. In order to reduce reservoir heterogeneity and modeling uncertainty, Jain et al. applied the idea of geology-engineering integration, combined with high-pressure physical properties, phase percolation data and geological information, to achieve optimal results for unconventional reservoir development
[4]. Batch operations on small platforms based on geology-engineering integration technology can effectively save time, increase well drainage area, and ultimately maximize the interests of oil and gas company [5]. Wu et al. introduced the concept of integration of geology and engineering into the Sichuan Basin and the surrounding marine shale gas regions to realize the economic and effective development of shale gas [6]. The integration method of geology and engineering has guided the optimization and adjustment of the entire life cycle of shale gas reservoirs in southern Sichuan, effectively increased the production of single well and EUR, and supported the economic development of shale gas [7].

Based on the geological model, combined with rock mechanics experimental analysis and dynamic rock mechanics parameter from logging, considering the influence of factors such as structure, faults and natural fractures, establishes three-dimensional geomechanics model to simulate the influence of deep reservoir diagenetic environment and three-dimensional stress. Combined with geological model and 3D geomechanical model, simulating the true 3D artificial fracture network based on 3D attribute cube, finally to establish the geology-engineering integration model of Wei $\mathrm{AH}$ platform, and provide theoretical basis and technical

\footnotetext{
* Corresponding author: Jiangh_jx@enpc.com.cn
} 
support for optimization of fracturing methods and parameters.

\section{Brief geological introduction of Wei AH platform and establishment of geological model}

\subsection{Brief geological introduction of Wei $\mathrm{AH}$ platform}

The whole area of well Wei A has a trend of steep in north, gentle in south, shallow in west and deep in south. The northwest part is the southeast wing of the Weiyuan structure, which is distributed in a monoclinic manner. The burial depth of the target layer is relatively shallow as a whole. The burial depth of the Wei A is between $2100 \mathrm{~m}$ and $3900 \mathrm{~m}$, with a trend of shallow in north and deep in south, and the overall pattern is similar to the structural map. The surface structure of Wei A area is relatively simple. The strata is steep in the north and gentle in the south. There are no faults. The faults of the Xujiahe Formation, the bottom of the Upper Permian, and the bottom of the Sinian are relatively developed, but the scale of faults is relatively still small. The fault's strike of Wei A area is multi-directional: nearly north-south, northeast, north-west, and nearly east-west.

Most of the blocks in the Wei A well area are buried within $3600 \mathrm{~m}$, which is a favorable area for shale gas exploration. The vertical change of the TOC in Weiyuan area is similar and have a good contrast relationship. They all have the characteristics of gradually getting better from top to bottom. Among them, the Longyi 11 layer has the highest abundance of organic matter, which is the most beneficial section for shale gas enrichment. Another condition for shale gas accumulation is the maturity, which is mostly within the range of thermal gasgenerating window (Ro is from $0.5 \%$ to $2.5 \%$ ).

\subsection{Establishment of geological model for Wei AH platform}

Integrating the seismic interpretation, logging interpretation and the fine gas reservoir description of the Wei AH platform, the three-dimensional geological model was established, including a natural fracture prediction model based on ant tracking, which provides a geological basis for the establishment of the geologyengineering integration model.

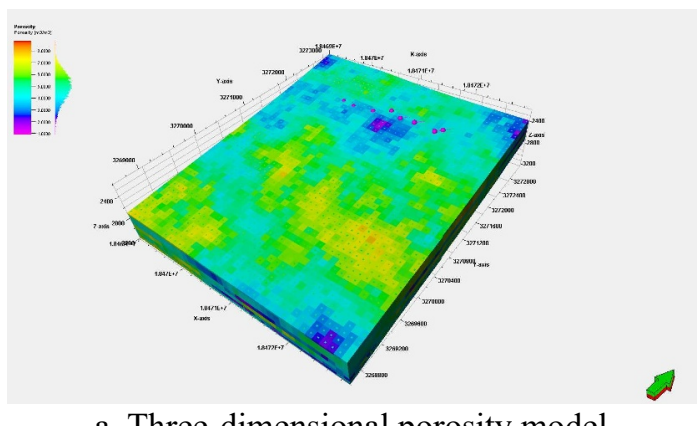

a. Three-dimensional porosity model

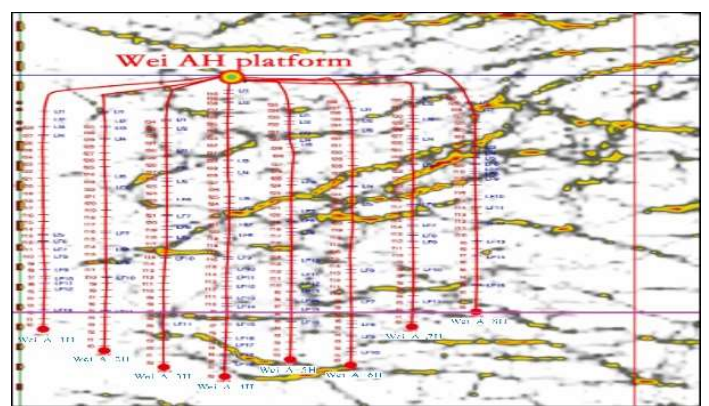

b. The natural fracture prediction model

Figure 1. The three-dimensional geological model of Wei AH platform

\section{Establishment of geomechanical model for Wei AH platform}

Based on logging interpretation, rock mechanics and acoustic experiments, the one-dimensional rock mechanics profile of the Wei $\mathrm{AH}$ well area was established. Synthesized geological model and onedimensional rock mechanics profile of the Wei $\mathrm{AH}$ platform, the deep diagenesis theory was used to consider the influence of overlying, basement and surrounding rock, to simulate diagenetic process by applying circumferential horizontal stress and build the geomechanical model of the Wei AH platform, which provides a geomechanical basis for drilling and fracturing design.

\subsection{Initialization of 3D geomechanical model}

On the basis of single-well triaxial rock mechanics experiment and acoustic emission experiment data analysis, based on density, longitudinal wave and other logging curve sequences, the Young's modulus, Poisson's ratio, and geomechanical parameters such as horizontal stress are calculated. Through the fitting and correction of the dynamic and static parameters of reservoir rock mechanics, the one-dimensional geomechanical profile models of three wells were obtained.

Loaded the rock mechanics profile of the 8 wells of the Wei AH platform into the logging interpretation attribute body of the geological model, referring to the attribute body modeling process of porosity and permeability, and using Kriging interpolation to generate three-dimensional attribute body of rock mechanical parameters such as Young's modulus, Poisson's ratio, etc., finally to provide a basic model of geomechanical parameters for regional stress simulation.

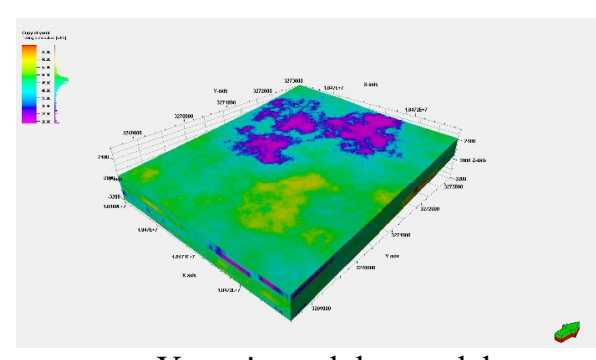

a. Young's modulus model 


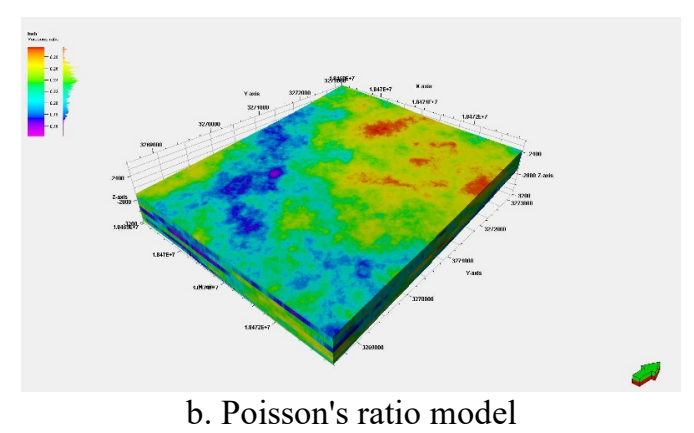

Figure 2. Initialization of 3D geomechanical model

\subsection{The deep diagenetic theory and modeling}

Different from the gas reservoir production model, the geomechanical model not only needs to consider the gas reservoir, but also the overlying rock, the underlying rock or the rock below the gas reservoir, as well as the lateral or surrounding rock that provides stress boundary conditions. Finite element simulation is widely used in stress analysis of conventional engineering and geomechanics, while finite difference simulation is used in fluid flow analysis. Connected above two types of analysis, it can be described and simulated the coupling characteristics when geomechanics and fluid flow change over time.

In order to simulate the real diagenetic environment of the deep reservoir, the vector displacement field and the continuous displacement compatibility equations were selected, the overlying, basement and surrounding rocks were added to the periphery of the study area, and the equivalent elastoplastic material was filled (Fig. 3a). The equivalent steel plate constitutive equation was selected to eliminate the stress concentration on boundary, and steel plate was added in the peripheral area (Fig. 3b).

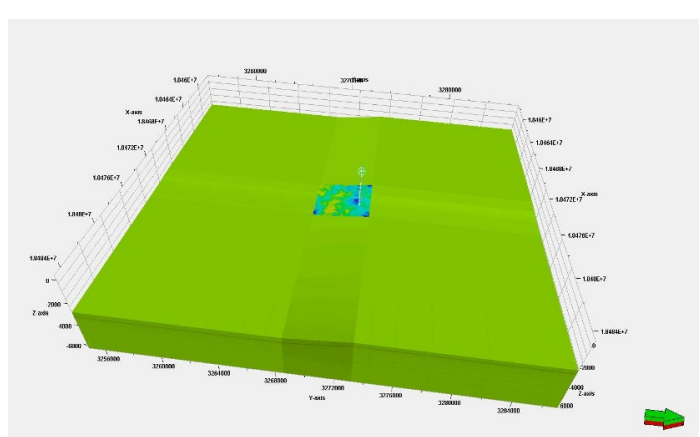

a. To add overlying, basement and surrounding rocks

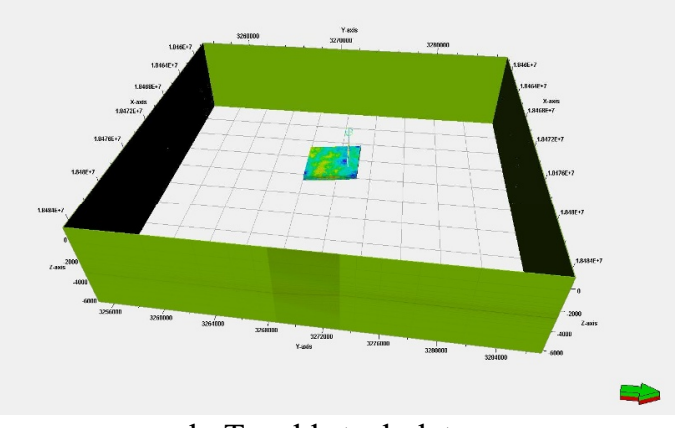

b. To add steel plate

Figure 3. Simulation of deep diagenetic environment
To load the circumferential stress, it is need to determine the current regional stress state and to load the vertical stress according to the rock filling condition, then we should integrate fluid simulation and stress simulation methods, starting from the fracturing changes predicted by the reservoir numerical simulation, to evaluate stress and strain changes caused by production and injection operations. Endowing certain temperature, pore pressure and three-dimensional in-situ stress to the reservoir formation, to simulate the rock diagenesis process, finally the current in-situ stress field distribution model of the research block can be obtained.

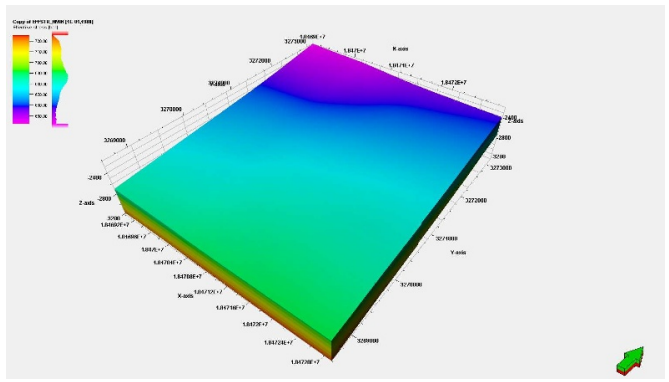

a. Minimum horizontal stress

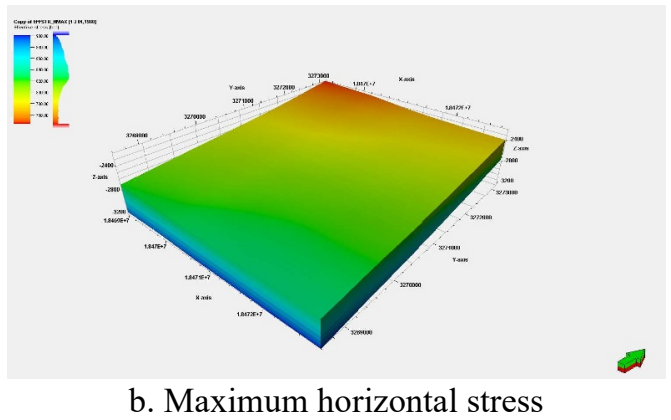

Figure 4. Horizontal stress distribution map of Wei AH platform

\section{Establishment of drilling and completion integration model of Wei $\mathrm{AH}$ platform}

On the basis of geological and engineering dual sweet spot selection, synthesizing geological and geomechanical model to design well trajectory and fracturing optimization.

\subsection{Integrated design for well trajectory}

Optimizing the design of horizontal well spacing and row spacing by using the reservoir numerical simulation method. Because the integrated model of fracturing optimization design was considered, therefore, the integrated well trajectory design and fracturing optimization design were actually the process carried out simultaneously and verified by mutual matching. On the basis of geological model, geomechanical model and integrated fracturing optimization design model, geological targets were described and optimized by geological sweet spots such as porosity and gas saturation, and the geological targets were finally set in the 
geological model. It has laid the foundation for the well trajectory design.

While visualizing the well trajectory design, the engineering sweet spots of horizontal staged fracturing should be considered, especially the geomechanical sweet spots in the geomechanical model. The vertical well section-curved section-vertical well section (or curve section) design could be completed based on the optimization of compressibility evaluation parameters. After setting the well trajectory, using the Ellipsoid of uncertainty method (EOU) to complete the well trajectory correction.

\subsection{Integrated design for fracturing optimization}

The advantage of integrated fracturing design is to realize seamless connection of data from geophysical prospecting, geology, drilling, completion and reservoir numerical simulation, and to combine three-dimensional attribute models, natural fractures, geomechanics, and even microseismic fracture in hydraulic fracturing simulation.

The artificial fracture simulation adopts the UFM model, which can simulate the expansion, deformation and fluid flow of fractures in the complex fracture network, it can also consider the fluid flow in the fracture network and the elastic deformation of fractures. The UFM model can simulate the interaction between hydraulic fractures and pre-existing natural fractures, as well as the expansion of non-planar complex fracturing modes caused by branch fractures which produced by the intersection of hydraulic fractures and natural fractures. In addition to the interaction between hydraulic fractures and natural fractures, UFM also considers the interaction between adjacent hydraulic fracture branches by calculating the "stress shadow" effect of adjacent fractures on each fracture.

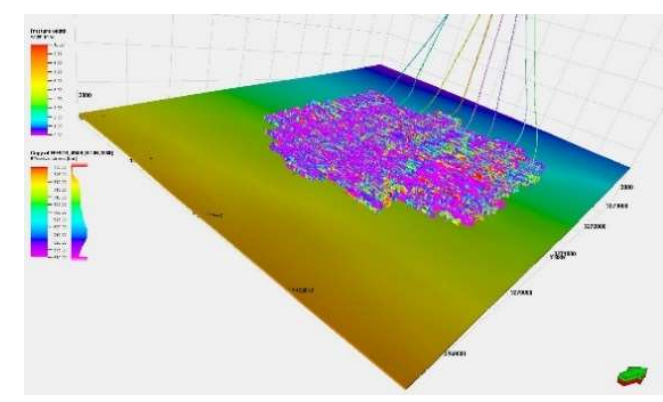

a. 3D view of fracturing model

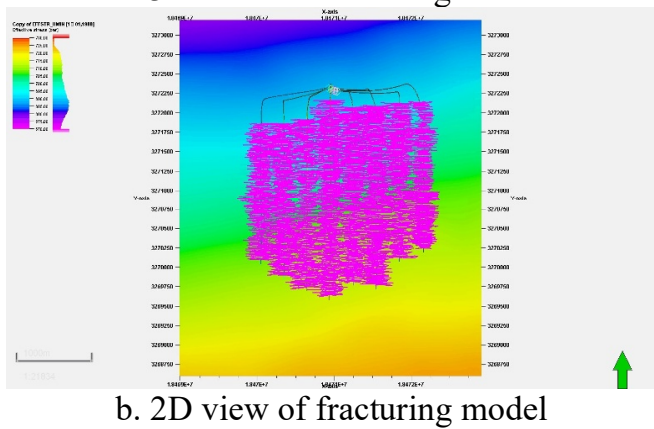

Figure 5. Integrated fracturing model of Wei AH platform

\section{Conclusion}

First, the Weiyuan shale gas block has dense reservoirs and complex geological engineering conditions. It is necessary to apply the comprehensive application of geophysical, geological and geomechanical parameters, and introduce the concept of geology-engineering integration to effectively complete the optimization design of drilling, completion and mining method.

Secondly, the geology-engineering integration model of shale reservoirs needs to consider the deep diagenesis process to establish a geomechanical model. Based on the geological model and geomechanical model, the drilling and completion design were completed by synthesizing well trajectory design, fracturing optimization design model and reservoir numerical simulation model. And all the models are nested and mutually verified.

\section{Acknowledgments}

This work was financially supported by the National Natural Science Foundation of China (No. 51504042, No.51604043); Subitem of National Science and Technology Major Project (2016ZX05048-001-04-LH); Key Project of Sichuan Provincial Education Department Foundation(18ZA0063).

\section{References}

1. Guang Yang, Weizhi Tian, Jiang Lv, et al. Petrological Characteristics of Shale Gas Reservoirs in Long11 Sub-member of Longmaxi Formation in W202 Block of Weiyuan Structure, J. Special Oil \& Gas Reservoirs, 2021, 28(2): 34-40.

2. Naizheng Liu, et al. Practice and prospect of geologyengineering integration technology in the efficient development of shale gas in Weiyuan block, J. China Petroleum Exploration, 2018, 23(2), 59-68.

3. Fuhao Zhao, Weian Huang, Rui Yong, et al. Research and application status of geology-engineering integration, J. Oil drilling and production technology, 2021, 43(2): 131-138.

4. A. K. Jain, H. Ahmad, P. K. Choudhary, et al. Integration of Geological, PVT and SCAL Data to Reduce Reservoir Uncertainty of an Unconventional Field in Kuwait, paper IPTC 18008 presented at the International Petroleum Technology Conference, Kuala Lumpur, Malaysia, December 10-12, 2014,

5. Yixiang Lyu. A Project Mode in Developing Oil and Gas on Artificial Islands: Cases Study of EPC, Batch Operation and Extended Reach Drilling, paper SPE 188663 presented at the Abu Dhabi International Petroleum Exhibition \& Conference, Abu Dhabi, UAE, November 13-16, 2017.

6. Wu Qi, Liang Xing, Xian Chenggang, et al. Geoscience-to-Production Integration Ensures Effective and Efficient South China Marine Shale Gas Development, J. China Petroleum Exploration, 2015, 20(4): 1-23. 
7. Chen Gengsheng, Wu Jianfa, Liu Yong, et al. Geology-engineering integration key technologies for ten billion cubic meters of shale gas productivity construction in the Southern Sichuan Basin, J. Natural Gas Industry, 2021, 41(1): 72-82. 